\title{
Perceptual experience and posttest improvements in perceptual accuracy and consistency
}

\author{
Jeffrey B. Wagman, Dawn M. MCBride, and Amanda J. Trefzger \\ Illinois State University, Normal, Illinois
}

\begin{abstract}
Two experiments investigated the relationship between perceptual experience (during practice) and posttest improvements in perceptual accuracy and consistency. Experiment 1 investigated the potential relationship between how often knowledge of results $(\mathrm{KR})$ is provided during a practice session and posttest improvements in perceptual accuracy. Experiment 2 investigated the potential relationship between how often practice (PR) is provided during a practice session and posttest improvements in perceptual consistency. The results of both experiments are consistent with previous findings that perceptual accuracy improves only when practice includes $\mathrm{KR}$ and that perceptual consistency improves regardless of whether practice includes KR. In addition, the results showed that although there is a relationship between how often KR is provided during a practice session and posttest improvements in perceptual accuracy, there is no relationship between how often PR is provided during a practice session and posttest improvements in consistency.
\end{abstract}

Perceptual learning is the improvement of perceptual skill with practice; it is the process of becoming a better perceiver (Epstein, 1967; Fahle \& Poggio, 2002; E. J. Gibson, 1969; E. J. Gibson \& Pick, 2000; Goldstone, 1998). Although repeated experience in a perceptual task is required for perceptual learning to occur, different kinds of perceptual experience lead to different kinds of improvement in perceptual skill. The consistency of a perceptual report is the degree to which stimuli can be differentiated on a perceptual scale. Consistency of perceptual reports improves with repeated experience in a perceptual task regardless of whether that experience includes any explicit feedback about task performance (Fahle \& Edelman, 1993; E. J. Gibson, 1969; E. J. Gibson \& Bergman, 1954; Karni \& Sagi, 1993; Wagman, Shockley, Riley, \& Turvey, 2001). Given that repeated perceptual experience itself is sufficient to improve perceptual consistency, such improvements have been termed "genuine perceptual learning" (E. J. Gibson, 1969; J. J. Gibson \& E. J. Gibson, 1955).

The accuracy of a perceptual report is the degree to which the perceived stimulus value matches the actual stimulus value. Unlike consistency of perceptual reports, the accuracy of perceptual reports improves only when experience in a perceptual task includes explicit feedback about task performance (E. J. Gibson, 1969; E. J. Gibson \& Bergman, 1954; Quinn, Palmer, \& Slater, 1999). However, such feedback must provide information about how a perceived stimulus value compares with the actual stimulus value (Withagen \& Michaels, 2005). This type of feedback (knowledge of results, or KR) allows the perceiver to calibrate the perceptual scale with respect to the actual metrical values and thus brings about improvements in accuracy (E. J. Gibson, 1969; Wagman et al., 2001).
In general, experiments on perceptual learning include a pretest, a practice session (or sessions), and a posttest. Typically, when KR is provided in such experiments, it is provided after every trial in a practice session (see Fahle, Edelman, \& Poggio, 1995; E. J. Gibson \& Bergman, 1954; Jacobs, Michaels, \& Runeson, 2000; Michaels \& de Vries, 1998; Quinn et al., 1999; Wagman et al., 2001; Withagen \& Michaels, 2004, 2005). Although such work has shown that providing KR after every practice trial is sufficient to improve posttest perceptual accuracy, it has not shown that doing so is necessary. That is, the common practice of providing $\mathrm{KR}$ on every practice trial has precluded an investigation of any potential relationship between how often $K R$ is provided during a practice session and posttest improvements in perceptual accuracy. This relationship may provide insight into the role that KR plays in bringing about improvements in perceptual accuracy (see Wagman, Carello, Schmidt, \& Turvey, 2006; Withagen \& Michaels, 2005).

Although there seem to be very few, if any, investigations of the relationship between how often KR is provided during a practice session and posttest improvements in perceptual accuracy, there have been a number of investigations of this relationship with respect to posttest improvements in movement accuracy (for a review, see Schmidt \& Lee, 2005). In these experiments, participants perform movement tasks, such as tapping (Lee, White, \& Carnahan, 1990), moving a lever (Winstein \& Schmidt, 1990), or moving a slide along a track (Sparrow \& Summers, 1992), and they are then provided with KR on some portion of the practice trials. Many of these investigations have shown that posttest movement accuracy improves as much when KR is provided on a portion of practice trials as when KR is provided on every practice trial (Lee et al., 1990; Sparrow \& Summers, 1992;

J. B.Wagman, jbwagma@ilstu.edu 
Winstein \& Schmidt, 1990; for a review, see Schmidt \& Lee, 2005). Presumably, however, there is a minimum embedding of KR in a practice session below which there would be little or no posttest improvement in perceptual accuracy.

In motor learning, it has been proposed that KR has an "informational function" because it provides not only information about whether the performance is accurate but also a prescription for how to improve performance on subsequent trials (Salmoni, Schmidt, \& Walter, 1984; for a review, see Schmidt \& Lee, 2005). If KR plays a similar role in perceptual learning (see Withagen \& Michaels, 2005), then we should expect posttest improvements in perceptual accuracy even when KR is provided on a portion of practice trials. The purpose of Experiment 1 was to investigate this hypothesis as well as to investigate the potential relationship between how often KR is provided during a practice session and posttest improvements in perceptual accuracy.

\section{EXPERIMENT 1}

In Experiment 1, we investigated posttest changes in accuracy and consistency of the perceived length of an occluded handheld object when KR was provided on every practice trial $(1.0 \mathrm{KR})$, on every other practice trial (.50 $\mathrm{KR}$ ), on every fourth practice trial (.25 KR), and on every eighth practice trial (.125 KR), and when KR was not provided on any practice trials ( $0 \mathrm{KR})$.

We expected posttest improvements in perceptual accuracy only when KR was provided during practice. Although we expected such improvements even when KR was provided on a portion of practice trials, we did not expect such improvements to be completely independent of how often KR was provided during practice. That is, we expected a relationship between how often KR was provided during a practice session and posttest improvements in perceptual accuracy. In addition, we expected posttest improvements in perceptual consistency regardless of whether KR was provided during practice and regardless of how often KR was provided during practice. That is, we did not expect a relationship between how often KR was provided during a practice session and posttest improvements in perceptual consistency.

\section{Method}

\section{Participants}

Seventy students (61 women and 9 men) from Illinois State University participated in this experiment in fulfillment of an extra credit option in their psychology courses.

\section{Materials and Apparatus}

Twelve T-shaped wooden objects were used as stimuli. Each object consisted of two wooden rods (both $1.2 \mathrm{~cm}$ in diameter) configured as a stem and a crossbar. One end of the stem was affixed to the center of the crossbar to create a $\mathrm{T}$ shape that was symmetric about its longitudinal axis. The stems ranged in length from 15 to $70 \mathrm{~cm}$ in $5-\mathrm{cm}$ increments, and the crossbars ranged in width from 10 to $65 \mathrm{~cm}$ in $5-\mathrm{cm}$ increments. The objects were constructed so that each object (stem plus crossbar) consisted of the same amount of wood (i.e., the $65-\mathrm{cm}$ crossbar was attached to the $15-\mathrm{cm}$ stem, the $60-\mathrm{cm}$ crossbar was attached to the $20-\mathrm{cm}$ stem, etc.). Therefore, the mass of each object was held constant ( $55 \mathrm{~g}$ each).
The objects were constructed in this way for two related reasons. First, doing so served as a safeguard against a ceiling effect in the length perception task. Previous research had shown that participants are generally quite accurate and consistent in perceiving the lengths of objects via dynamic touch (Turvey, Burton, Amazeen, Butwill, \& Carello, 1998; Wagman et al., 2001; for reviews, see Carello \& Turvey, 2004, and Turvey, 1996). Eliminating the relationship between mass and length forces participants to search for more subtle scaling relationships between the felt properties of a given object and its length (e.g., the relationship between mass distribution of the object and its length). Second, doing so maximized the likelihood that any perceptual learning (in particular, any improvement in accuracy) exhibited in the posttest would be due to a genuine rescaling of perceptual reports to the felt properties of the rod (and not merely a decision to modify posttest perceptual reports by a fixed amount). The T-shaped objects with $20-, 30-, 40-$, $50-, 60-$, and $70-\mathrm{cm}$ stems were designated the test objects. The T-shaped objects with 15-, 25-, 35-, 45-, 55-, and 65-cm stems were designated the practice objects.

Participants sat in a right-handed student desk and placed their right forearm onto the desk. They slid their right hand through a curtain, which occluded both the hand and the handheld object. The report apparatus consisted of an adjustable flag along a $240-\mathrm{cm}$ wooden track at a height of $70 \mathrm{~cm}$. Participants adjusted the distance of the flag toward or away from them using a pulley system such that the distance between the flag and the zero point of the apparatus corresponded to the perceived length of the stem of the object. A tape measure secured to the floor allowed the experimenter to read reports of perceived length (in centimeters, measured from the wrist of the participant). The tape measure was not visible to the participant.

\section{Design and Procedure}

The experiment consisted of three sets of trials: a pretest, a practice session, and a posttest. Each set consisted of 24 trials, for a total of 72 trials per participant. The 70 participants were randomly assigned to one of five KR conditions: $1.0, .50, .25, .125$, and $0 \mathrm{KR}$. The number preceding KR refers to the proportion of KR trials in the practice session.

Pretest. Participants were seated and placed their right arm on the armrest. They placed their right hand through the curtain in such a way that their right wrist was aligned with the zero point of the report apparatus. They were handed one of the test objects by the experimenter, and they grasped the stem firmly as close to the bottom of the object (i.e., the end of the stem that was not affixed to the crossbar) as possible. They attempted to gain an impression of the length of the stem of the object by wielding the object about their wrist and then reported this perceived length by adjusting the distance of the flag as described above. Wielding was not restricted in any way, except that participants were instructed to refrain from touching the occlusion curtain with the handheld object. Participants could wield each object as long as was necessary in order to achieve an impression of the length of the stem, and they were allowed to continually adjust the report apparatus until they were satisfied with their perceptual report. After reporting the perceived length, the participant returned the flag to the zero point of the apparatus and was handed the next object. Each participant wielded the six test objects four times each, and the order of objects was randomized. Participants did not see the objects at any time before or during the experiment. The procedure for the pretest was the same for all participants in each of the five conditions.

Practice session. Following the pretest, participants in all five conditions participated in a practice session using the practice objects. The practice session consisted of two types of trials - no knowledge of results (no-KR) and KR. A no-KR trial proceeded exactly as in the pretest. A KR trial proceeded in the same way, except that after the participant made a perceptual report, the experimenter repositioned the flag so that the distance of the flag corresponded to the actual length of the stem of the object. On these trials, participants were encouraged to wield the object while looking at the repositioned flag after KR had been provided (cf. Withagen \& Michaels, 2004, Experiment 2). 
Participants in the $1.0 \mathrm{KR}$ condition performed KR trials on every trial (24 out of 24 trials) and did not perform any no-KR trials. Participants in the $.50 \mathrm{KR}$ condition performed KR trials on every other trial (12 out of 24 trials) and performed no-KR trials on the remaining trials. Participants in the $.25 \mathrm{KR}$ condition performed KR trials on every fourth trial ( 6 out of 24 trials) and performed no-KR trials on the remaining trials. Participants in the $.125 \mathrm{KR}$ condition performed KR trials on every eighth trial ( 3 out of 24 trials) and performed no-KR trials on the remaining trials. Participants in the $0 \mathrm{KR}$ condition did not perform any KR trials ( 0 out of 24 trials) and performed only no-KR trials.

As in the pretest, participants could wield each object as long as was necessary in order to achieve an impression of its length, and they were allowed to continually adjust the report apparatus until they were satisfied with their perceptual report. Within a given condition, the six practice objects were wielded four times each and the order of presentation was randomized. As a result, the object wielded on a given KR trial was randomly determined.

Posttest. The procedure for the posttest was identical to the procedure for the pretest. As in the pretest, the procedure was the same for all participants.

\section{Results and Discussion}

\section{Accuracy Data}

Percent absolute error. The overall accuracy of the perceptual reports was assessed with percent absolute error $(\% \mathrm{AE})$ :

$$
\% \mathrm{AE}=\left[\left(\sum\left|x_{i}-D\right| / n\right) / D\right] * 100,
$$

where $D$ is the actual magnitude, $x_{i}$ is the perceived magnitude on trial $i$, and $n$ is the number of trials on which judgments are made. AE quantifies the absolute difference between a perceptual report and the actual stimulus value and thus indexes the accuracy with which a perceptual scale is applied (E. J. Gibson, 1969). Expressing $\mathrm{AE}$ as a percentage of the mean perceived length (\%AE) provides a normalized measure of accuracy of perceptual reports (Wagman et al., 2001).

A 5 (proportion of KR trials) $\times 2$ (pretest vs. posttest) ANOVA was conducted on $\%$ AE values. A main effect of test showed that \%AE decreased from pretest $(29.7 \%)$ to posttest $(23.8 \%)\left[F(1,65)=36.52, M S_{\mathrm{e}}=33.67, p<\right.$ $.01]$, and a main effect of proportion of KR trials showed that $\% \mathrm{AE}$ differed across $\mathrm{KR}$ conditions $[F(4,65)=8.01$, $\left.M S_{\mathrm{e}}=112.45, p<.01\right] .{ }^{1}$ An interaction of these variables showed that the changes in $\% \mathrm{AE}$ from pretest to posttest depended on the proportion of KR trials provided during the practice session $\left[F(4,65)=2.91, M S_{\mathrm{e}}=33.67, p<.05\right]$. A series of follow-up paired-samples $t$ tests (using a Bonferroni correction) revealed that $\% \mathrm{AE}$ decreased from pretest to posttest in the $1.0 \mathrm{KR}$ condition [pretest, $26.0 \%$; posttest, $17.7 \% ; t(13)=6.81, p<.01]$, in the $.50 \mathrm{KR}$ condition [pretest, $27.5 \%$; posttest, $18.1 \%$; $t(13)=5.44, p<.01$ ], and in the $.25 \mathrm{KR}$ condition [pretest, $29.3 \%$; posttest, $22.0 \%$; $t(13)=4.11, p<.01]$, but not in the other conditions (all other $p \mathrm{~s}>.05)$ (see Figure 1). To determine whether \%AE decreased by the same amount from pretest to posttest in the $1.0 \mathrm{KR}, .50 \mathrm{KR}$, and $.25 \mathrm{KR}$ conditions, we calculated difference scores for each participant (i.e., \%AE in pretest minus \% AE in posttest) and compared these scores in a one-way ANOVA. This analysis showed that these values differed across $\mathrm{KR}$ conditions $[F(4,69)=2.91, p<.05]$, but follow-up tests revealed no differences among the scores in the $1.0 \mathrm{KR}, .50 \mathrm{KR}$, and $.25 \mathrm{KR}$ conditions (all $p \mathrm{~s}>$ .05) (see Figure 1). Power analyses were conducted with the $\mathrm{G}^{*}$ Power program (Faul, Erdfelder, Lang, \& Buchner, 2007). Effect sizes for power analyses were estimated from conditions showing a significant effect. All effect sizes were estimated to be "large" (Keppel \& Wickens, 2004). Power was estimated to be greater than .99 for all tests.

Slope and intercept. Although \% AE is a useful measure of the overall accuracy of perceptual reports (E. J. Gibson, 1969; Wagman et al., 2001), it does not differentiate between inaccuracy due to scaling error and inaccuracy due to offset error. To assess the potential improvement of perceptual reports with respect to these sources of inaccuracy, we computed regression lines with perceived length as the dependent variable and actual length as the independent variable for each participant in both the pretest and the posttest. ${ }^{2}$ We then performed parallel 5 (proportion of KR trials) $\times 2$ (pretest vs. posttest) ANOVAs on the slopes and intercepts of the regression lines (see Withagen \& Michaels, 2004).

When the ANOVA was performed on the intercept values, a main effect of test revealed that intercept decreased from pretest $(22.3)$ to posttest $(16.4)\left[F(1,65)=35.65, M S_{\mathrm{e}}=\right.$ $34.42, p<.01]$, and a main effect of proportion of KR trials showed that intercept differed across KR conditions $\left[F(4,65)=4.07, M S_{\mathrm{e}}=128.82, p<.01\right]$. An interaction of these variables showed that the changes in intercept from pretest to posttest depended on the proportion of KR trials provided during the practice session $[F(4,65)=6.71$, $\left.M S_{\mathrm{e}}=34.42, p<.01\right]$. A series of follow-up paired-samples $t$ tests (using a Bonferroni correction) revealed that intercept decreased from pretest to posttest in the $1.0 \mathrm{KR}$ condition [pretest, 22.3; posttest, 8.2; $t(13)=5.33, p<.01$ ] and in the $.50 \mathrm{KR}$ condition [pretest, 20.6; posttest, $10.8 ; t(13)=4.47$, $p<.01]$ but not in the other conditions (all other $p \mathrm{~s}>.05$ ) (see Figure 2). To determine whether the intercept decreased by the same amount from pretest to posttest in the $1.0 \mathrm{KR}$

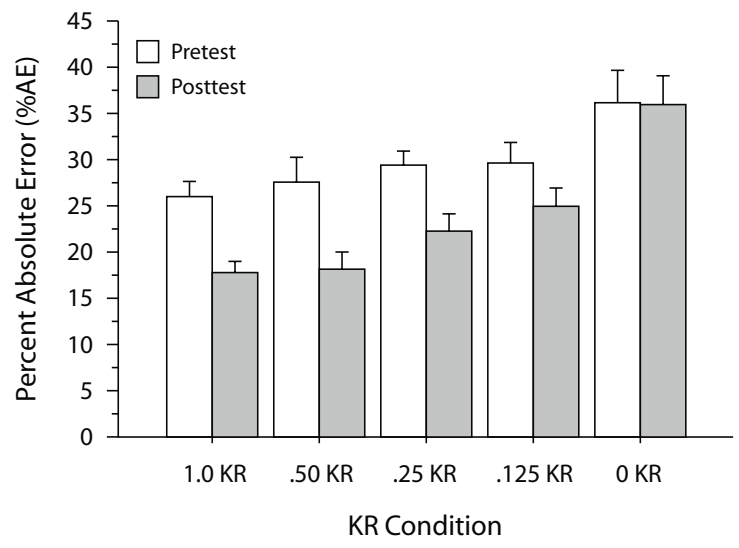

Figure 1. Percent absolute error (\%AE) decreased from pretest to posttest when KR was provided on every practice trial (1.0 KR), when KR was provided on every other practice trial (.50 KR), and when KR was provided on every fourth practice trial (.25 KR). There was no change from pretest to posttest in the other conditions. The error bars represent standard errors. 
and the $.50 \mathrm{KR}$ conditions, we calculated difference scores for each participant (i.e., intercept in pretest minus intercept in posttest) and compared these scores in a one-way ANOVA. This analysis showed that these values differed in the different $\mathrm{KR}$ conditions $[F(4,69)=6.71, p<.01]$, but follow-up tests revealed no differences between the scores in the $1.0 \mathrm{KR}$ and $.50 \mathrm{KR}$ conditions (all $p \mathrm{~s}>.05$ ) (see Figure 2). Using the method described above, power was estimated to be greater than .99 for all tests.

When the ANOVA was performed on the slope values, a main effect of test revealed that slope increased from pretest $(0.42)$ to posttest $(0.48)\left[F(1,65)=40.84, M S_{\mathrm{e}}=.02\right.$, $p<.01]$. An interaction of test and proportion of KR trials showed that the changes in slope from pretest to posttest depended on the proportion of KR trials provided during the practice session $\left[F(4,65)=4.62, M S_{\mathrm{e}}=.02, p<\right.$ $.01]$. The main effect of proportion of KR trials was not significant $\left[F(4,65)=1.87, M S_{\mathrm{e}}=.07\right.$, n.s. $]$. A series of follow-up paired-samples $t$ tests (using a Bonferroni correction) revealed that slope increased from pretest to posttest in the $1.0 \mathrm{KR}$ condition [pretest, 0.43 ; posttest, 0.74 ; $t(13)=-7.57, p<.01]$ and in the $.50 \mathrm{KR}$ condition [pretest, 0.42 ; posttest, $0.69 ; t(13)=-3.58, p<.01$ ] but not in the other conditions $(p s>.05)$. To determine whether slope increased by the same amount from pretest to posttest in the $1.0 \mathrm{KR}$ and the $.50 \mathrm{KR}$ conditions, we calculated difference scores for each participant (i.e., slope in posttest minus slope in pretest) and compared these scores in a oneway ANOVA. This analysis showed that these values differed in the different $\mathrm{KR}$ conditions $[F(4,69)=4.62, p<$ $.01]$, but follow-up tests revealed no difference between the scores in the $1.0 \mathrm{KR}$ and the $.50 \mathrm{KR}$ conditions $(p>.05)$ (see Figure 3). Using the method described above, power was estimated to be greater than .99 for all tests.

\section{Consistency Data}

Percent variable error. Consistency of perceptual reports was quantified by percent variable error $(\% \mathrm{VE})$ :

$$
\% \mathrm{VE}=\left[\frac{\sqrt{\sum\left(x_{i}-M\right)^{2} / n}}{M}\right] * 100,
$$

where $M$ is the mean perceptual report for a given rod length, and $x_{i}, i$, and $n$ are defined as above. VE quantifies the range of perceptual reports each time that stimulus is encountered and thus indexes the precision with which a perceptual scale is applied (E. J. Gibson, 1953, 1969; cf. Wagman et al., 2001). Expressing VE as a percentage of the mean perceived length $(\% \mathrm{VE})$ provides a normalized measure of consistency of perceptual reports (cf. Carello, Thuot, Anderson, \& Turvey, 1999; Carello, Thuot, \& Turvey, 2000) and makes it a comparable measure to \%AE.

A 5 (proportion of KR trials) $\times 2$ (pretest vs. posttest) ANOVA was conducted on $\% \mathrm{VE}$ values. A main effect of test showed that $\% \mathrm{VE}$ decreased from pretest $(6.4 \%)$ to posttest $(5.5 \%)\left[F(1,65)=12.52, M S_{\mathrm{e}}=2.65, p<.01\right]$. The main effect of proportion of KR trials was nonsignificant $\left[F(4,65)=1.65, M S_{\mathrm{e}}=2.65\right.$, n.s. $]$, as was the interaction of test and proportion of KR trials $[F(4,65)=$

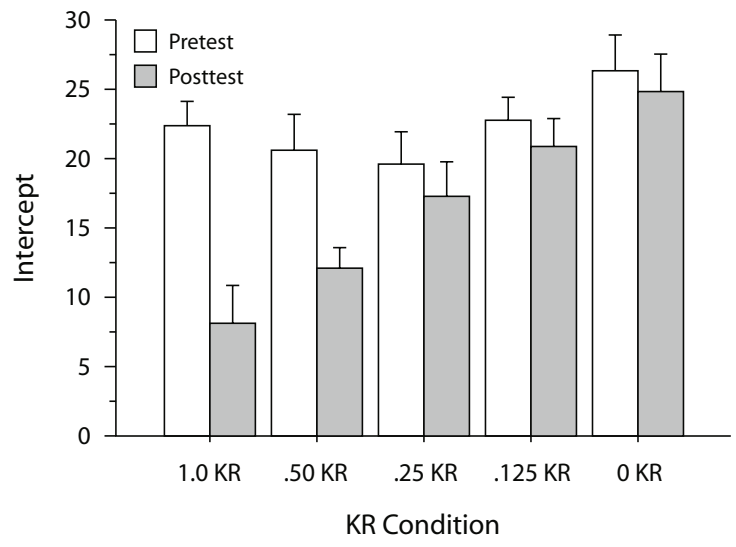

Figure 2. Intercept increased from pretest to posttest when KR was provided on every practice trial (1.0 KR) and when KR was provided on every other practice trial $(.50 \mathrm{KR})$. There was no change from pretest to posttest in the other conditions. The error bars represent standard errors.

$1.82, M S_{\mathrm{e}}=7.58$, n.s.], suggesting that the decrease in $\% \mathrm{VE}$ from pretest to posttest did not differ across KR conditions. Using the method described above, power was estimated to be greater than .99 for all tests.

In summary, the results of Experiment 1 are consistent with previous research showing posttest improvements in perceptual accuracy only when KR is provided during practice (E. J. Gibson, 1969; Wagman et al., 2001; Withagen \& Michaels, 2005). Moreover, they support the hypothesis that although such improvements occur even when KR is provided on a portion of practice trials, they are not independent of how often KR is provided during practice. That is, the results show a relationship between how often KR is provided during a practice session and posttest improvements in perceptual accuracy. The exact nature of this relationship (how often KR must be provided

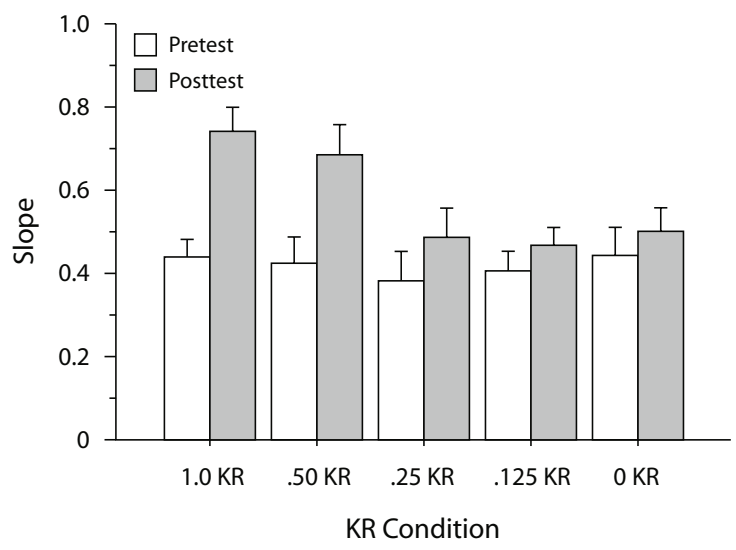

Figure 3. Slope decreased from pretest to posttest when KR was provided on every practice trial $(1.0 \mathrm{KR})$ and when $\mathrm{KR}$ was provided on every other practice trial (.50 KR). There was no change from pretest to posttest in the other conditions. The error bars represent standard errors. 
to bring about such improvements) seems to be measuredependent; whereas all three measures $(\% \mathrm{AE}$, intercept, and slope) showed that perceptual accuracy improved in the 1.0 KR and .50 KR conditions, \%AE also showed improvement in the $.25 \mathrm{KR}$ condition.

The results of Experiment 1 are also consistent with previous research showing posttest improvements in perceptual consistency, regardless of whether KR is provided during practice. Moreover, they support the hypothesis that such improvements occur regardless of how often KR is provided during practice. That is, the results show no relationship between how often KR is provided during a practice session and posttest improvements in perceptual consistency.

\section{EXPERIMENT 2}

Whereas the purpose of Experiment 1 was to investigate the relationship between how often $\mathrm{KR}$ is provided during a practice session and posttest improvement in perceptual accuracy, the purpose of Experiment 2 was to investigate the relationship between how often practice (without knowledge of results) (PR) is provided during a practice session and posttest improvement in perceptual consistency. This relationship should provide insight into the role that PR plays in bringing about such improvements.

Specifically, in Experiment 2, we investigated posttest changes in accuracy and consistency of the perceived length of an occluded handheld object when PR was provided on every trial during a practice session (1.0 PR), on every other trial during a practice session (.50 PR), on every fourth trial during a practice session (.25 PR), on every eighth trial during a practice session (.125 PR), and when PR was not provided during a practice session (0 PR). We did not expect posttest improvements in perceptual accuracy in any condition. However, we expected posttest improvements in perceptual consistency regardless of how often PR was provided during a practice session.

\section{Method}

\section{Participants}

Seventy students (56 women and 14 men) from Illinois State University participated in this experiment in fulfillment of an extra credit option in their psychology courses.

\section{Materials and Apparatus}

The materials and apparatus used in Experiment 2 were identical to those used in Experiment 1.

\section{Design and Procedure}

The experiment consisted of three sets of trials: a pretest, a practice session, and a posttest. Each set consisted of 24 trials, for a total of 72 trials per participant. The 70 participants were randomly assigned to one of five conditions: 1.0 PR, .50 PR, .25 PR, .125 PR, and 0 PR. The number preceding PR refers to the proportion of practice trials in the practice session.

Pretest. The pretest of Experiment 2 was identical to that of Experiment 1 .

Practice session. The practice session consisted of two types of trials: practice (PR) and no-practice (no-PR). A PR trial proceeded exactly as during the pretest but used the practice objects. A no-PR trial did not involve wielding a T-shaped object. Instead, the experimenter read the name of a U.S. state, and the participant attempted to recall the capital city of that state. The purpose of each of the no-PR trials was to engage the participant in an unrelated (filler) task that would be comparable in length to a PR trial.

Participants in the 1.0 PR condition performed PR trials on every trial (24 out of 24 trials) and did not perform any no-PR trials. Participants in the $.50 \mathrm{PR}$ condition performed PR trials on every other trial (12 out of 24 trials) and performed no-PR trials on the remaining trials. Participants in the .25 PR condition performed PR trials on every 4 th trial ( 6 out of 24 trials) and performed no-PR trials on the remaining trials. Participants in the .125 PR condition performed PR trials on every 8 th trial ( 3 out of 24 trials) and performed no-PR trials on the remaining trials. Participants in the $0 \mathrm{PR}$ condition did not perform any PR trials ( 0 out of 24 trials) and performed only no-PR trials.

On PR trials, participants could wield each object as long as was necessary for them to achieve an impression of length and were allowed to continually adjust the report apparatus until they were satisfied with their perceptual report. The order of presentation of the six practice objects was randomized, and no object was presented more than four times to a given participant. On no-PR trials, participants could take as much time as necessary to report the capital of the U.S. state (or to report that they did not know the capital of that state). The state read on a given no-PR trial was randomly determined, and no state was presented more than once to a given participant

Posttest. The procedure for the posttest was identical to the procedure for the pretest. As in the pretest, the procedure was the same for all participants.

\section{Results and Discussion}

We conducted the same analyses in Experiment 2 as in Experiment 1.

\section{Accuracy Data}

Percent absolute error. A 5 (proportion of PR trials) $\times$ 2 (pretest vs. posttest) ANOVA on \%AE values revealed no main effect of test $\left[F(1,65)=0.05, M S_{\mathrm{e}}=22.95\right.$, n.s.], no main effect of proportion of PR trials $[F(4,65)=0.27$, $M S_{\mathrm{e}}=102.37$, n.s.], and no interaction of these variables $\left[F(4,65)=0.31, M S_{\mathrm{e}}=22.95\right.$, n.s.]. As in Experiment 1, power analyses were conducted with the $G *$ Power program (Faul et al., 2007). Effect sizes were estimated to be large, given the significant effects found in Experiment 1 as well as the significant effect of test on $\% \mathrm{VE}$ (see below). Power was estimated to be greater than .95 for all tests.

Slope and intercept. ${ }^{3}$ A 5 (proportion of PR trials) $\times 2$ (pretest vs. posttest) ANOVA on intercept values revealed no main effect of test $\left[F(1,65)=0.23, M S_{\mathrm{e}}=25.32\right.$, n.s. $]$, no main effect of proportion of PR trials $[F(4,65)=1.43$, $M S_{\mathrm{e}}=117.96$, n.s.], and no interaction of these variables $\left[F(4,65)=0.61, M S_{\mathrm{e}}=25.32\right.$, n.s. $]$. Power was estimated to be greater than .95 for all tests.

A 5 (proportion of PR trials) $\times 2$ (pretest vs. posttest) ANOVA on slope values revealed a main effect of proportion of PR trials - slope differed across the PR conditions $\left[F(4,65)=2.64, M S_{\mathrm{e}}=.09, p<.05\right]$; but there was no main effect of test $\left[F(1,65)=2.82, M S_{\mathrm{e}}=.02\right.$, n.s. $]$, and there was no interaction of these variables $[F(4,65)=$ $1.46, M S_{\mathrm{e}}=.02$, n.s.]. ${ }^{4}$ Power was estimated to be greater than .95 for all tests.

\section{Consistency Data}

Percent variable error. A 5 (proportion of PR trials) $\times$ 2 (pretest vs. posttest) ANOVA on \%VE values revealed a main effect of test: \%VE decreased from pretest (6.5\%) to posttest $(5.0 \%)\left[F(1,65)=33.88, M S_{\mathrm{e}}=2.12, p<.01\right]$. The 
main effect of proportion of PR trials was not significant $\left[F(4,65)=0.69, M S_{\mathrm{e}}=4.43\right.$, n.s. $]$, and neither was the interaction of test and proportion of PR trials $[F(4,65)=0.61$, $M S_{\mathrm{e}}=2.12$, n.s.], suggesting that the decrease in $\% \mathrm{VE}$ from pretest to posttest did not differ across PR conditions. Power was estimated to be greater than .95 for all tests.

In summary, the results of Experiment 2 are consistent with previous research showing posttest improvements in perceptual accuracy only when KR is provided during practice and posttest improvements in perceptual consistency regardless of whether $\mathrm{KR}$ is provided during practice (E. J. Gibson, 1969; Wagman et al., 2001; Withagen \& Michaels, 2005). Moreover, they not only support the hypothesis that posttest improvements in perceptual consistency occur regardless of how often PR is provided during a practice session, but also show that such improvements occur even when PR is not provided during a practice session. That is, the results show no relationship between how often $\mathrm{PR}$ is provided during a practice session and posttest improvements in perceptual consistency.

\section{GENERAL DISCUSSION}

Two experiments investigated how perceptual experience during practice influences posttest improvements in perceptual accuracy and consistency. In particular, in Experiment 1, we investigated the potential relationship between how often $\mathrm{KR}$ is provided during a practice session and posttest improvements in perceptual accuracy, and in Experiment 2, we investigated the potential relationship between how often PR is provided during a practice session and posttest improvements in perceptual consistency. The results of both experiments are consistent with previous findings showing that (1) posttest perceptual accuracy improves only when practice includes KR (Wagman et al., 2001; Withagen \& Michaels, 2005; for a review, see E. J. Gibson, 1969) and (2) posttest perceptual consistency improves regardless of whether practice includes KR (Wagman et al., 2006; Wagman et al., 2001; for a review, see E. J. Gibson, 1969).

In addition the results of Experiment 1 support the hypothesis that although improvements in perceptual accuracy occur even when KR is provided on a portion of practice trials, such improvements are not independent of how often KR is provided during practice. That is, there seems to be a relationship between how often KR is provided during a practice session and posttest improvements in perceptual accuracy. The results of Experiment 2 support the hypothesis that improvements in perceptual consistency occur regardless of how often (and whether) PR is provided during a practice session. That is, there seems to be little or no relationship between how often PR is provided during a practice session and posttest improvements in consistency.

\section{Knowledge of Results and Improvements in Perceptual Accuracy}

Interestingly, the nature of this relationship between how often $\mathrm{KR}$ is provided during a practice session and posttest improvements in perceptual accuracy seems to be measure dependent (\%AE, slope, or intercept). This is likely due to differences in the level of analysis of the error measures.
As stated above, \%AE is a composite measure that quantifies the overall error in perceptual reports without regard to the source of the error (offset or scaling error). Intercept and slope quantify the sources of error - offset and scaling error, respectively. Therefore, whereas \%AE may be sensitive to smaller amounts of error, intercept and slope provide a more detailed description of that error. Regardless of such differences, the results provide converging evidence that, in this task, not only does perceptual accuracy improve when KR is provided on every other trial in a practice session, it does so to the same degree as when KR is provided on every trial in a practice session.

Such results are consistent with work investigating posttest improvements in movement accuracy (Lee et al., 1990; Sparrow \& Summers, 1992; Winstein \& Schmidt, 1990; for a review, see Schmidt \& Lee, 2005) and suggest that, perhaps, KR plays an informational role in perceptual learning similar to that which it plays in motor learning. In both processes, KR provided during practice guides the perceiveractor toward completing a goal-directed behavior not only by informing the perceiver-actor about the correctness of the response but also by providing the perceiver-actor with a prescription for how to improve on subsequent trials (Lee et al., 1990; Winstein \& Schmidt, 1990; for a review, see Withagen \& Michaels, 2005). The results of the two experiments reported here suggest that in perceptual learning, as in motor learning, KR serves this informational function equally well when it is presented on some portion of practice trials and when it is presented on every practice trial. However, the results also demonstrate that in this perceptual learning task, the informational value of $\mathrm{KR}$ is diminished when it is presented less often than on every other practice trial. This may be the minimum embedding of KR in a practice session required in this task for the perceiver to "get the range of a set of stimuli" (E. J. Gibson, 1953) or calibrate his/her perceptual scale with respect to the set of stimulus values (E. J. Gibson, 1969; Jacobs, Runeson, \& Michaels, 2001; Wagman et al., 2001).

Future research may provide a more detailed understanding of the relationship between how much KR is provided during practice and posttest improvements in perceptual accuracy. For example, it is not necessarily clear whether the particular relationship uncovered here is related to the perceptual system investigated, the experimental task, or any of the specific experimental conditions used in the experiments - particularly the total number of practice trials, the relative (and absolute) number of practice trials during which $\mathrm{KR}$ is provided, the distribution of $\mathrm{KR}$ throughout the practice trials, and the length of time between pretest and practice and between practice and posttest. Although these factors have been shown to play roles in motor learning (see Schmidt \& Lee, 2005), the effect of these factors on perceptual learning is less clear. For example, several studies have shown that when there is a long delay (e.g., 24-48 h) between practice session and posttest, there are greater improvements in posttest movement accuracy when $\mathrm{KR}$ is provided on a portion of practice trials than when KR is provided on every practice trial (Schmidt, Young, Swinnen, \& Shapiro, 1989; Wulf \& Schmidt, 1989). When there is little or no delay between practice session 
and posttest, the results are more consistent with those of the present experiment (i.e., there is little or no difference between posttest improvements in each case).

\section{Practice and Improvements in Perceptual Consistency}

Experiment 1 revealed that posttest perceptual consistency improved to the same degree regardless of whether KR was provided (and regardless of how often KR was provided) during a practice session (see Wagman et al., 2006; Wagman et al., 2001). Such improvements in the ability to differentiate values within a perceptual scale seem to reflect the intrinsic tuning of perceptual systems (Fahle \& Edelman, 1993; E. J. Gibson, 1969; E. J. Gibson \& Bergman, 1954; Karni \& Sagi, 1993; Wagman et al., 2001). Such results are consistent with work in motor learning suggesting that trials during which $\mathrm{KR}$ is not provided (i.e., PR trials) still contribute to the learning process in some way, though perhaps in a way that is different from practice trials where KR is provided (see Schmidt \& Lee, 2005).

More surprisingly, however, Experiment 2 revealed that posttest perceptual consistency improved to the same degree regardless of whether (and how often) PR was provided during the practice trials. It seems that not only do posttest improvements in perceptual consistency occur independently of whether KR is provided during a practice session, such improvements occur regardless of whether $\mathrm{PR}$ is provided during a practice session. In Experiment 2, perceptual consistency improved even when there was no explicit practice provided during a practice session. However, although such improvements seem to be independent of explicit practice provided during a practice session, they are not necessarily independent of experience in performing the task. Rather, it seems that the minimal perceptual experience in the pretest (and posttest) was sufficient for perceivers to develop an improved ability to differentiate the objects within a perceptual scale. Moreover, such minimal experience was sufficient to improve this ability as much as repeatedly providing this perceptual experience on every trial in a practice session.

It seems that merely providing perceptual reports for a few of the objects in the pretest provided "anchors" within an emerging perceptual scale such that the other objects could then be perceived with respect to (and differentiated within) this scale. Such findings not only reinforce the notion that the development of a better differentiated perceptual scale does not require experiences over and above performance of the perceptual task itself (E. J. Gibson \& Bergman, 1954; J. J. Gibson \& E. J. Gibson, 1955; Wagman et al., 2001), but also suggest that such development requires only relatively little experience in the perceptual task. How little experience is required may depend on a number of factors, such as the complexity of the perceptual task or the variation among the objects or events being perceived (see Jacobs et al., 2001). This may be an additional topic for future research.

\section{Speculations on a Potential Link Between Perceptual Learning and Motor Learning}

The possibility that KR seems to serve the same informational function in motor learning as in perceptual learning (or a similar one) is consistent with fundamental conceptual and empirical links between perception and motor behavior (see Clark, 1997; J. J. Gibson, 1979; Hommel, Müsseler, Aschersleben, \& Prinz, 2001; Mechsner, 2004; Turvey \& Shaw, 1999) and between perceptual learning and motor learning (see Hecht, Vogt, \& Prinz, 2001; Mechsner, Kerzel, Knoblich, \& Prinz, 2001; Repp \& Knoblich, 2004). One speculative proposal regarding the link between perceptual learning and motor learning from J. J. Gibson's (1979) perspective on perception and Bernstein's (1967) perspective on motor control is offered in what follows (see Michaels \& de Vries, 1998; Runeson, Juslin, \& Olsson, 2000).

From Bernstein's perspective, the primary issue in motor learning is that of uncovering task-specific control units from an excessive number of biomechanical degrees of freedom (Bernstein, 1967; Latash \& Turvey, 1996; Turvey, 1990). From J. J. Gibson's perspective, the primary issue in perceptual learning is the uncovering of task-specific detection units from an excessive stimulation flux (J. J. Gibson, 1979; Wagman \& Miller, 2003). In each case, the perceiver-actor is faced with uncovering task-specific units and calibrating those units with respect to environmental spatiotemporal patterns (a required movement pattern in the case of motor learning and a range of stimulus values in the case of perceptual learning). Perhaps KR plays a similar informational role in each process by facilitating the calibration in each case.

\section{Concluding Comments}

The results of the present two experiments suggest that whereas there is a relationship between how often KR is provided during a practice session and posttest improvements in perceptual accuracy, there is no relationship between how often PR is provided during a practice session and posttest improvements in perceptual consistency. These results have theoretical implications for the understanding of the roles of KR and PR in perceptual learning and may have practical implications for the development of training techniques for individuals who require specific perceptual skills, such as pilots, surgeons, or military personnel (see McCarley, Kramer, Wickens, Vidoni, \& Boot, 2004).

\section{AUTHOR NOTE}

We thank Kyriakos Tsiappoutas for help with data collection. Correspondence concerning this article should be addressed to J. B. Wagman, Department of Psychology, Campus Box 4620, Illinois State University, Normal, IL 61790 (e-mail: jbwagma@ilstu.edu).

\section{REFERENCES}

BernsteIn, N. A. (1967). The co-ordination and regulation of movements. London: Pergamon.

Carello, C., Thuot, S., Anderson, K. L., \& Turvey, M. T. (1999). Perceiving the sweet spot. Perception, 28, 307-320.

Carello, C., Thuot, S., \& Turvey, M. T. (2000). Aging and the perception of a racket's sweet spot. Human Movement Science, 19, 1-20.

Carello, C., \& Turvey, M. T. (2004). Physics and the psychology of the muscle sense. Current Directions in Psychological Science, 13, 25-28.

Clark, A. (1997). Being there: Putting brain, body, and world together again. Cambridge, MA: MIT Press.

Epstein, W. (1967). Varieties of perceptual learning. New York: McGraw-Hill.

FAHLE, M., \& Edelman, S. (1993). Long-term learning in vernier acuity: Effects of stimulus orientation, range and of feedback. Vision Research, 33, 397-412. 
Fahle, M., Edelman, S., \& Poggio, T. (1995). Fast perceptual learning in hyperacuity. Vision Research, 35, 3003-3013.

Fahle, M., \& Poggio, T. (Eds.) (2002). Perceptual learning. Cambridge, MA: MIT Press.

Faul, F., Erdfelder, E., Lang, A.-G., \& Buchner, A. (2007). G*Power 3: A flexible statistical power analysis program for the social, behavioral, and biomedical sciences. Behavior Research Methods, 39, 175-191.

Gibson, E. J. (1953). Improvement in perceptual judgments as a function of controlled practice or training. Psychological Bulletin, 50, 401-431.

Gibson, E. J. (1969). Principles of perceptual learning and development. New York: Appleton-Century-Crofts.

GiBson, E. J., \& BERGMAN, R. (1954). The effect of training on absolute estimation of distance over the ground. Journal of Experimental Psychology, 48, 473-482.

Gibson, E. J., \& Pick, A. D. (2000). An ecological approach to perceptual learning and development. Oxford: Oxford University Press.

GiBson, J. J. (1979). The ecological approach to visual perception. Boston: Houghton Mifflin.

Gibson, J. J., \& Gibson, E. J. (1955). Perceptual learning: Differentiation or enrichment? Psychological Review, 62, 32-41.

Goldstone, R. L. (1998). Perceptual learning. Annual Review of Psychology, 49, 585-612.

Hecht, H., Vogt, S., \& Prinz, W. (2001). Motor learning enhances perceptual judgment: A case for action-perception transfer. Psychological Research, 65, 3-14.

Hommel, B., Müsseler, J., Aschersleben, G., \& Prinz, W. (2001). The Theory of Event Coding (TEC): A framework for perception and action planning. Behavioral \& Brain Sciences, 24, 849-937.

Jacobs, D. M., Michaels, C. F., \& Runeson, S. (2000). Learning to perceive the relative mass of colliding balls: The effects of ratio scaling and feedback. Perception \& Psychophysics, 62, 1332-1340.

Jacobs, D. M., Runeson, S., \& Michaels, C. F. (2001). Learning to visually perceive the relative mass of colliding balls in globally and locally constrained task ecologies. Journal of Experimental Psychology: Human Perception \& Performance, 27, 1019-1038.

KARNI, A., \& SAGI, D. (1993). The time course of learning a visual skill. Nature, 365, 250-252.

KePPEL, G., \& Wickens, T. D. (2004). Design and analysis: A researcher's handbook. Englewood Cliffs, NJ: Prentice Hall.

Latash, M. L., \& Turvey, M. T. (EDs.) (1996). Dexterity and its development. Mahwah, NJ: Erlbaum.

Lee, T. D., White, M. A., \& Carnahan, H. (1990). On the role of knowledge of results in motor learning: Exploring the guidance hypothesis. Journal of Motor Behavior, 22, 191-208.

McCarley, J. S., Kramer, A. F., Wickens, C. D., Vidoni, E. D., \& Bоot, W. R. (2004). Visual skills in airport-security screening. Psychological Science, 15, 302-306.

MeCHSNER, F. (2004). A psychological approach to human voluntary movements. Journal of Motor Behavior, 36, 355-370.

Mechsner, F., Kerzel, D., Knoblich, G., \& Prinz, W. (2001). Perceptual basis of bimanual coordination. Nature, 414, 69-73.

Michaels, C. F., \& DE VRies, M. M. (1998). Higher order and lower order variables in the visual perception of relative pulling force. Journal of Experimental Psychology: Human Perception \& Performance, 24, 526-546.

QuinN, P. C., Palmer, V., \& Slater, A. M. (1999). Identification of gender in domestic-cat faces with and without training: Perceptual learning of a natural categorization task. Perception, 28, 749-763.

Repp, B. H., \& KNoblich, G. (2004). Perceiving action identity: How pianists recognize their own performances. Psychological Science, 15, 604-609.

Runeson, S., Juslin, P., \& Olsson, H. (2000). Visual perception of dynamic properties: Cue heuristics versus direct-perceptual competence. Psychological Review, 107, 525-555.

Salmoni, A. W., Schmidt, R. A., \& Walter, C. B. (1984). Knowledge of results and motor learning: A review and critical reappraisal. Psychological Bulletin, 95, 355-386.

Schmidt, R. A., \& LeE, T. D. (2005). Motor control and learning: A behavioral emphasis (4th ed.). Champaign, IL: Human Kinetics.

Schmidt, R. A., Young, D. E., Swinnen, S., \& Shapiro, D. C. (1989).
Summary knowledge of results for skill acquisition: Support for the guidance hypothesis. Journal of Experimental Psychology: Learning, Memory, \& Cognition, 15, 352-359.

Sparrow, W. A., \& Summers, J. J. (1992). Performance on trials without knowledge of results $(\mathrm{KR})$ in reduced relative frequency presentations of KR. Journal of Motor Behavior, 24, 197-209.

Turvey, M. T. (1990). Coordination. American Psychologist, 45, 938-953.

Turvey, M. T. (1996). Dynamic touch. American Psychologist, 51, 1134-1152.

Turvey, M. T., Burton, G., Amazeen, E. L., Butwill, M., \& Carello, C. (1998). Perceiving the width and height of a handheld object by dynamic touch. Journal of Experimental Psychology: Human Perception \& Performance, 24, 35-48.

Turvey, M. T., \& Shaw, R. E. (1999). Ecological foundations of cognition: I. Symmetry and specificity of animal-environment systems. Journal of Consciousness Studies, 6, 95-110.

Wagman, J. B., Carello, C., Schmidt, R. C., \& Turvey, M. T. (2006). Is perceptual learning unimodal? Manuscript submitted for publication.

Wagman, J. B., \& Miller, D. B. (2003). Nested reciprocities: The organism-environment system in perception-action and development. Developmental Psychobiology, 42, 317-334.

Wagman, J. B., Shockley, K., Riley, M. A., \& Turvey, M. T. (2001). Attunement, calibration, and exploration in fast haptic perceptual learning. Journal of Motor Behavior, 33, 323-327.

Winstein, C. J., \& SCHMidT, R. A. (1990). Reduced frequency of knowledge of results enhances motor skill learning. Journal of Experimental Psychology: Learning, Memory, \& Cognition, 16, 677-691.

Withagen, R., \& Michaels, C. F. (2004). Transfer of calibration in length perception by dynamic touch. Perception \& Psychophysics, 66, 1282-1292.

Withagen, R., \& Michaels, C. F. (2005). The role of feedback information for calibration and attunement in perceiving length by dynamic touch. Journal of Experimental Psychology: Human Perception \& Performance, 31, 1379-1390.

WULF, G., \& SCHMIDT, R. A. (1989). The learning of generalized motor programs: Reducing the relative frequency of knowledge of results enhances memory. Journal of Experimental Psychology: Learning, Memory, \& Cognition, 15, 748-757.

\section{NOTES}

1. Inspection of Figure 1 suggests that in the pretest, $\% \mathrm{AE}$ differs in the different KR conditions. Indeed, this is the case $[F(4,69)=2.62, p<$ $.05]$. However, this difference seems to have been due to the influence of 1 participant in the $0 \mathrm{KR}$ condition whose pretest $\% \mathrm{AE}(67.5 \%)$ was nearly double that of the mean value in this condition $(36.2 \%)$. When this participant is removed from the analysis, this difference becomes nonsignificant. However, such preexisting differences in \%AE have little, if any, bearing on the present hypotheses, which focus on changes (or lack thereof) from pretest to posttest. This is especially so given that no significant differences were observed in \%AE from pretest to posttest in the $0 \mathrm{KR}$ condition.

2 . The mean explained variances of the regression lines relating perceived length to actual length $\left(r^{2}\right)$ ranged from .78 to .89 in the pretest and from .87 and .92 in the posttest, suggesting that in each case, perceived length was a linear function of actual length

3. As in Experiment 1, we computed regression lines with perceived length as the dependent variable and actual length as the independent variable for each participant in both the pretest and the posttest. The mean explained variances of the regression lines relating perceived length to actual length $\left(r^{2}\right)$ ranged from .78 to .92 in the pretest and from .83 and .93 in the posttest, suggesting that in each case, perceived length was a linear function of actual length.

4. The difference in slope across the different PR conditions is likely due to preexisting differences in the scaling of perceived length to actual length among the groups of participants. As in Experiment 1, such differences have little, if any, bearing on the present hypotheses, which focus on changes (or lack thereof) from pretest to posttest.

(Manuscript received May 14, 2007; revision accepted for publication March 10, 2008.) 